$F(G)=V_{T}$, there exists an element $y$ of $G$ such that $F y=x^{\prime}$. Thus the distinct elements $x, y$ of $H$ are mapped by $F$ into the same element $x^{\prime}$ of $V_{T}$, contrary to condition (ii).

\title{
REFERENCES
}

1. Reinhold Baer, The subgroup of elements of finite order of an abelian group, Ann. of Math. vol. 37 (1936) pp. 766-781.

2. Hans Hahn, Über die nichtarchimedischen Grössensysteme, Sitz. der K. Akad. der Wiss., Math. Nat. Kl. vol. 116, Abt. IIa (1907) pp. 601-655.

3. M. Hausner and J. G. Wendel, Ordered vector spaces, Proc. Amer. Math. Soc. vol. 3 (1952) pp. 977-982.

4. Paul F. Conrad, Embedding theorems for abelian groups with valuations, Amer. J. Math. vol. 75 (1953) pp. 1-29. (Added in proof.)

The Johns Hopkins University

\section{A NOTE ON A RECENT RESULT IN SUMMABILITY THEORY}

\author{
C. F. MARTIN
}

In a recent paper ${ }^{1}$ by $A$. Mary Tropper, the following theorem is given:

THEOREM. In order that the regular ${ }^{2}$ normal ${ }^{3}$ matrix $A$ shall sum a bounded divergent sequence, it is sufficient that

(a) its unique reciprocal $B$ shall not be regular and

(b) there exists a normal matrix $Q$ with ${ }^{4}\|Q\|<\infty$ whose columns are all null sequences, such that the matrix $C=B Q$ has bounded columns and $\|C\|=\infty$.

The author points out that (a) is also a necessary condition, but does not prove the necessity of condition (b); the object of this note is to prove that condition (b) is necessary.

The proof given of the theorem quoted above holds if the $K_{r}$

Received by the editors January 5, 1954.

1 Proc. Amer. Math. Soc. vol. 4 (1953) pp. 671-677.

2 A regular matrix is one which satisfies $\lim _{n \rightarrow \infty} \sum_{k=1}^{\infty} a_{n, k} x_{k}=\lim _{k \rightarrow \infty} x_{k}$, whenever the latter limit exists.

${ }^{3}$ A normal matrix is a lower-semi matrix which has no zero elements in the leading diagonal. Such a matrix has a unique two-sided reciprocal; see e.g., R. G. Cooke, Infinite matrices and sequence spaces, Macmillan, 1950, p. 19.

${ }^{4}$ For an infinite matrix $M,\|M\| \equiv \sup _{n} \sum_{k}\left|m_{n, k}\right|$ is called the $K_{r}$ bound of the matrix: a matrix whose $K_{r}$ bound is finite is called a $K_{r}$ matrix (ibid. pp. 25, 29). 
matrix $Q$ of condition (b) is required to be a lower-semi, but not necessarily a normal, matrix; the necessity of condition (b) will first be proved under these conditions, and then it will be shown that the existence of a normal $K_{r}$ matrix $Q$ is also necessary.

Suppose, therefore, that $A$ sums the bounded divergent sequence $\left\{x_{i}\right\}$ to $x$. Without loss of generality, we may suppose $x=0$, since, if $x \neq 0, A$ sums the bounded divergent sequence $\left\{x_{i}-x\right\}$ to 0 .

Let $C^{(1)}$ be the normal matrix defined by

$$
c_{i, j}^{(1)}=x_{i}(j<i) ; \quad c_{i, i}^{(1)}=1 ; \quad c_{i, j}^{(1)}=0(j>i) .
$$

Any given column of $C^{(1)}$ is thus the sum of $\left\{x_{i}\right\}$ and a null sequence; hence its $A$-transform tends to 0 , i.e., the columns of $Q^{(1)} \equiv A C^{(1)}$ all form null sequences.

Also,

(1) $\left|q_{r, 8}^{(1)}\right|=\left|\sum_{t=1}^{r} a_{r, t} c_{t, 8}^{(1)}\right| \leqq \sup _{t}\left|c_{t, 8}^{(1)}\right| \sup _{r} \sum_{t=1}^{r}\left|a_{r, t}\right|<K \quad$ for all $r, s$.

Now a diagonal matrix $D$, whose diagonal elements are all either 0 or 1 , can be chosen in such a way that $D$ contains an infinity of 1 's and $Q=Q^{(1)} D$ is a $K_{r}$ matrix. For, to choose such a matrix, let the values of $n$ for which $d_{n}=1$ be denoted by $n_{1}, n_{2}, \cdots$, and suppose that $n_{1}, n_{2}, \cdots, n_{p}$ have been chosen such that

$$
\sum_{s=1}^{n_{\nu}}\left|q_{r, s}\right|=\sum_{k=1}^{\nu}\left|q_{r, n_{k}}^{(1)}\right| \leqq 2 K \text { whenever } r \geqq n_{\nu}(\nu=1,2, \cdots, p) .
$$

Since the columns of $Q^{(1)}$ tend to 0 , we may choose $n_{p+1}>n_{p}$ such that

$$
\sum_{k=1}^{p}\left|q_{r, n_{k}}^{(1)}\right| \leqq K \quad\left(r \geqq n_{p+1}\right),
$$

and hence, by (1),

$$
\sum_{s=1}^{n_{p+1}}\left|q_{r, s}\right| \leqq 2 K \quad\left(r \geqq n_{p+1}\right) .
$$

We may begin this construction by taking $n_{1}=1$, and it continues indefinitely, so that $D$ contains an infinity of 1 's.

We now show that with $D$ so defined, $\|Q\|<\infty$. Given any value of $r$, there exists $p$ such that $n_{p} \leqq r<n_{p+1}$; then

$$
\sum_{s=1}^{r}\left|q_{r, 8}\right|=\sum_{k=1}^{p}\left|q_{r, n_{k}}^{(1)}\right| \leqq 2 K,
$$


by (2), which is what is required.

Also, the columns of $Q$ form null sequences, since they are either columns of $Q^{(1)}$, or they consist entirely of zeros.

Again $C=B Q=B Q^{(1)} D=B A C^{(1)} D=C^{(1)} D$, where $B$ is the unique two-sided reciprocal of $A$. (The products are associative, since the matrices are all lower-semi.) Hence,

$$
\sum_{j=1}^{i}\left|c_{i, j}\right|=\sum_{k=1}^{\lambda_{i}}\left|c_{i, n_{k}}^{(1)}\right|
$$

where $\lambda_{i}$ is the number of 1 's in the first $i$ columns of $D$; thus

$$
\sum_{j=1}^{i}\left|c_{i, j}\right| \geqq\left(\lambda_{i}-1\right)\left|x_{i}\right| \text {. }
$$

But $\lim _{i \rightarrow \infty} \lambda_{i}=\infty$, and $\lim \sup _{i \rightarrow \infty}\left|x_{i}\right|>0$, since $\left\{x_{i}\right\}$ is divergent. Hence $\lim \sup _{i \rightarrow \infty} \sum_{j=1}^{i}\left|c_{i, j}\right|=\infty$, so that $\|C\|=\infty$.

Moreover, the columns of $C$ are bounded, for they are either columns of $C^{(1)}$, or they consist entirely of zeros.

This completes the proof in the case where $Q$ is required to be a lower-semi, but not necessarily normal, matrix.

To show that a normal matrix $Q$ also exists, we note that $C^{(1)}$ and $Q^{(1)}$ are normal matrices, and that $I-D$ is a diagonal matrix of 0 's and 1's whose nonzero elements occur only in those columns of $D$ which consist entirely of zeros. Hence $\bar{C} \equiv C+I-D=C^{(1)} D+I-D$ is a normal matrix with bounded columns, and $\|\bar{C}\|=\infty$.

The corresponding $Q$, i.e., $\bar{Q}=A \bar{C}$, is normal (being the product of normal matrices), and, since $\bar{Q}=A C^{(1)} D+A(I-D)=Q^{(1)} D$ $+A(I-D)$, we have $\|\bar{Q}\| \leqq\|Q\|+\|A\|<\infty$; also the columns of $\bar{Q}$, which are all either columns of $Q^{(1)}$ or columns of $A$, form null sequences.

Hence the existence of a normal matrix $Q$ with the required properties is proved.

Birkbeck College, University of London 